\title{
Gene Transfer of Pigment Epithelium-Derived Factor Suppresses Tumor Growth and Angiogenesis in a Hepatoblastoma Xenograft Model
}

\author{
MARYBETH BROWNE, VERONICA STELLMACH, MONA CORNWELL, CHUHAN CHUNG, JENNIFER A. DOLL, EUN-JIG LEE, \\ J. LARRY JAMESON, MARLETA REYNOLDS, RICCARDO A. SUPERINA, LISA P. ABRAMSON, AND SUSAN E. CRAWFORD
}

Department of Surgery [M.B., M.R., R.A.S., L.P.A.], Children's Memorial Hospital, Chicago, Illinois, 60614; Department of Pathology [V.S., M.C., J.A.D., S.E.C.], Department of Hepatology [C.C.], Department of Endocrinology [E.-J.L., J.L.J.], Northwestern University, Feinberg School of Medicine, Chicago, Illinois, 60611

\begin{abstract}
Normal hepatocytes express pigment epitheliumderived factor (PEDF), an endogenous antiangiogenic factor. We hypothesized that decreased PEDF expression may be one mechanism driving hepatoblastoma growth, and in vivo gene transfer of PEDF could suppress neovascularization and limit tumor growth. PEDF functional activity was determined in vitro using endothelial cell migration assays and in vivo using a subcutaneous tumor model. HUH-6 human hepatoblastoma tumors were treated with hybrid adenoviral/adeno-associated viral expression vectors for PEDF (HybPEDF, $n=4$ ) or $\beta$-galactosidase (Hyb- $\beta$ gal, $n=4$ ) daily for $4 \mathrm{~d}$. Mitotic figures, microvascular density (MVD), PEDF, and VEGF expression were assessed. Hyb-PEDF treatment inhibited in vivo tumor growth $(p<0.008)$ and decreased MVD $(p<0.001)$, the number of mitotic figures $(p<0.001)$, and VEGF expression when compared with Hyb- $\beta$ gal-treated tumors. HUH-6 expression of PEDF was dramatically reduced when cultured under hypoxic conditions and also when grown in vivo, and the addition of neutralizing anti-PEDF antibody increased the already high baseline angiogenic activity of the HUH-6 cell secretions in vitro $(p<0.04)$. PEDF is an important endogenous regulator of the liver vasculature. Augmenting intra-tumoral PEDF levels inhibits tumor growth by reducing angiogenesis and VEGF expression. Potent inhibitors of angiogenesis, such as PEDF, may be an effective alternative treatment for children with hepatoblastoma. (Pediatr Res 60: 282-287, 2006)
\end{abstract}

$\mathrm{H}$ epatoblastoma is the most common liver tumor in childhood, accounting for over $50 \%$ of liver masses and about $67 \%$ of malignant liver neoplasms (1). Like most solids tumors, hepatoblastoma requires neovascularization for growth and metastasis. Hepatoblastoma expresses several proangiogenic factors, including vascular endothelial growth factor (VEGF) (2), hepatocyte growth factor (HGF) (3), and IGF-II $(4,5)$, whose expression and/or signaling are modulated by hypoxia (6-10). Intratumoral hypoxia can shift the balance between endogenous proangiogenic and antiangiogenic factors, thus leading to the excessive neovascularization of the

Received October 6, 2005; accepted April 17, 2006.

Correspondence: Susan E. Crawford, M.D., Northwestern University, Department of Pathology, W127, 330 E. Chicago Ave., Chicago, IL 60611; e-mail: scrawford@ northwestern.edu

Presented at the 36th Annual Meeting of the American Pediatric Surgical Association (APSA), Phoenix, AZ, May 29 to June 1, 2005.

This work was supported by grant CA64239 from the National Institutes of Health.

DOI: 10.1203/01.pdr.0000232789.86632.91 growing tumor mass. Several studies have focused on characterizing hepatoblastoma-derived angiogenesis inducers, whereas very little is known about the endogenous inhibitors. Any decreased expression of inhibitory factors would also be expected to contribute to a proangiogenic environment, thus favoring hepatoblastoma growth.

Development of antiangiogenic therapies for cancer is an area of active research (11-13). Treatments targeting the endothelial cells may have the advantage in that their antitumor effect does not result in the acquired drug resistance that arises with conventional therapies that target the genetically unstable tumor cells, a current problem in some treatment regimens of hepatoblastoma $(14,15)$. In vivo models of hepatoblastoma have shown that therapeutic strategies focusing on blocking angiogenesis such as anti-VEGF antibody (16) and the angiogenic inhibitor TNP-470 (17) resulted in restricted tumor growth by inhibiting new vessel formation. Loss of critical endogenous inhibitors of angiogenesis may also contribute to the pro-angiogenic microenvironment in hepatoblastoma $(18,19)$; thus, replacement therapy may restore the angiogenic balance.

PEDF is a $50 \mathrm{kD}$ protein that is a potent inhibitor of angiogenesis (20) and is expressed at high levels in normal hepatocytes and liver parenchymal cells $(21,22)$. Purified recombinant PEDF protein has been shown to inhibit the effects of several inducers of angiogenesis on endothelial cells including, VEGF, aFGF (fibroblast growth factor), bFGF, IL-8, lysophosphatidic acid, and platelet-derived growth factor (20). In addition, expression of PEDF is sensitive to hypoxia in retinoblastoma cells (20) and some prostate-derived cells (23). We postulated that loss of PEDF in the hepatoblastoma tumor microenvironment leads to excessive neovascularization, and, thus, restoring the endogenous inhibitor PEDF by gene trans-

\footnotetext{
Abbreviations: Ad/AAV, adenoviral/adeno-associated virus; CM, conditioned media; hpf, high-powered field; HUH-6, human hepatoblastoma cell line; Hyb-ßgal, hybrid adenoviral/adeno-associated viral factor expressing $\beta$-galactosidase; Hyb-PEDF, hybrid adenoviral/adeno-associated viral vector expressing PEDF; ITR, inverted terminal repeat; MVD, microvessel density; PEDF, pigment epithelium-derived growth factor
} 
fer to an in vivo hepatoblastoma model will result in decreased tumor growth by blocking new vessel formation.

\section{MATERIALS AND METHODS}

Cell lines and collection of CM. The human hepatoblastoma cell line HUH-6 (RIKEN Cell Bank, Ibaraki, Japan) was maintained in Dulbecco's modified Eagle medium (DMEM; Invitrogen, Carlsbad, CA) supplemented with 10\% FCS (Hyclone Laboratories, Logan, UT), $100 \mathrm{IU} / \mathrm{mL}$ penicillin, and $100 \mathrm{IU} / \mathrm{mL}$ streptomycin (Cellgro, Mediatech, Herdon, VA) at $37^{\circ} \mathrm{C}$ in $5 \%$ $\mathrm{CO}_{2}$ atmosphere. Bovine adrenal vascular endothelial cells (VEC Technologies, Rensselaer, NY) were grown in DMEM with 5\% donor calf serum, 5\% FCS, $2 \mathrm{mM}$ glutamine, and $50 \mu \mathrm{g} / \mathrm{mL}$ endothelial cell mitogen (Biomedical Technologies, Stoughton, MA) at $37^{\circ} \mathrm{C}$ in $8 \% \mathrm{CO}_{2}$ atmosphere.

When HUH-6 cell cultures reached $85 \%$ confluence, the media were aspirated and cells were washed with PBS, fed with serum-free media, and incubated for $4 \mathrm{~h}$. The media were then aspirated, the cells washed a second time with PBS, and incubated in serum-free media for $48 \mathrm{~h}$, at which time the $\mathrm{CM}$ were collected. For hypoxic studies, cobalt chloride $(100 \mu \mathrm{M}$; Sigma Chemical Co.-Aldrich, St. Louis, MO) was added to the serum-free media during the collection period as described (23). CM were subjected to centrifugation $(1000 \times g, 5 \mathrm{~min})$ to remove particulate matter, the protease inhibitor phenyl-methane-sulfonyl fluoride (200 $\mu \mathrm{M}$; Sigma Chemical Co.-Aldrich) added, and media concentrated and dialyzed against PBS using $10 \mathrm{kD}$ cutoff centrifugal devices (Pall Life Sciences, East Hills, NY). Total protein concentrations were determined using Coomassie dye binding assays (Pierce, Rockford, IL).

PEDF immunoblot. Aliquots of $5 \mu \mathrm{g}$ total protein were separated by $10 \%$ SDS-PAGE, electro-blotted to Hybond-C membranes, (Amersham Pharmacia Biotech, Arlington Heights, IL), and PEDF detected using anti-PEDF antibody (20). Equal protein loading of samples was verified by Coomassie dye staining of identically loaded gels. Bio-Rad (Hercules, CA) prestained SDSPAGE low-range standards were used as molecular weight standards. Purified recombinant PEDF served as a positive control (20-30 ng/lane).

In vitro angiogenesis assay. Endothelial cells were used at passage 9-15 in the endothelial cell migration assay as described (24). Serum-free medium containing $0.1 \%$ BSA served as a control for background migration, and the angiogenic inducer VEGF served as a positive control. CM were tested at 20 $\mu \mathrm{g} / \mathrm{mL}$. Before use in the migration assay, antibodies were dialyzed against PBS. The concentrations of the proteins and antibodies used in the migration assays were as follows: VEGF, $500 \mathrm{pg} / \mathrm{mL}$; PEDF, $0.1 \mu \mathrm{g} / \mathrm{mL}$; anti-VEGF antibody, $20 \mu \mathrm{g} / \mathrm{mL}$; and anti-PEDF antibody, $1 \mu \mathrm{g} / \mathrm{mL}$. Results were reported as the number of endothelial cells migrated per $10 \mathrm{hpf}$. Controls demonstrating that proteins and neutralizing antibodies had the expected activity were conducted but are not shown. Media were assayed in quadruplicate and each experiment was performed a minimum of two times.

Production of Ad/AAV hybrid vector. A replication-deficient Ad/AAV hybrid vector was constructed in which the AAV- ITR flanks the transgene cassette. An adenoviral transfer plasmid (25) carrying left-hand ITR and packaging sequence $(\Psi)$ was used to generate this hybrid transfer plasmid. AAV-ITR-flanked multiple cloning sequences were inserted adjacent to the adenoviral left-end 393 base pairs of adenoviral transfer plasmid, resulting in the generation of backbone Ad/AAV hybrid transfer plasmid. A cassette containing either a histidine tagged human PEDF cDNA or E. coli $\beta$-galactosidase gene driven by the human cytomegalovirus promoter was inserted into multiple cloning site of hybrid transfer plasmid. The hybrid vectors carrying PEDF (Hyb-PEDF) or $\beta$-galactosidase (Hyb- $\beta$ gal) were generated, amplified, purified, and titrated as described (25). AAV-ITR sequences were obtained from pSUB201, an AAV transfer plasmid kindly provided by Dr. Samulski, University of North Carolina.

Animal model and administration of treatment. HUH-6 cells were grown to $85 \%$ confluence, harvested by trypsinization, counted in the presence of trypan blue to determine viability, washed, and resuspended in sterile PBS at a concentration of $3.0 \times 10^{7}$ viable cells $/ \mathrm{mL}$. The Institutional Animal Care and Use Committee of Northwestern University approved all animal experimental procedures. Four to six week female athymic mice (NCI, Bethesda, MD) were housed in a barrier facility and were acclimated to the environment for at least $48 \mathrm{~h}$ before use. Hepatoblastomas were induced by injecting $3.0 \times$ $10^{6}$ HUH-6 cells subcutaneously into the right hind flank of each mouse. Tumors were allowed to grow to $5 \mathrm{~mm}$ in diameter before initiation of treatment. The mice were randomly selected to receive intratumoral injections of $10^{7}$ infectious viral particles/dose of Hyb-PEDF $(n=4)$ or Hyb- $\beta$ gal $(n=$ 4). An additional four mice with tumors did not receive any treatment. The untreated mice were compared with the Hyb- $\beta$ gal animals to verify that the Hyb- $\beta$ gal did not inhibit nor enhance tumor growth. The animals were treated daily for $4 \mathrm{~d}$ and then killed on $\mathrm{d} 7$.
Tumor harvest and immunohistochemistry. The subcutaneous tumors were harvested, weighed, measured, and tumor volume calculated using the formula length $\times$ width $(2) \times 0.52$ as described (26). Portions of the tumor tissue were washed five times in sterile PBS, minced, and incubated in serum-free media for $48 \mathrm{~h}$ and CM collected as above. The remainder of the tumor tissue was formalin-fixed and paraffin embedded for histologic examination.

Histologic sections of tumor tissue were stained for PEDF, MVD, and VEGF using an affinity purified anti-PEDF antibody (20), anti-factor VIII related antigen antibody (R \& D Systems, Minneapolis, MN), and anti-VEGF antibody ( $\mathrm{R}$ \& D Systems), respectively. Sections of tissue were dried at $42^{\circ} \mathrm{C}$ for $24 \mathrm{~h}$ and deparaffinized, incubated at room temperature with primary antibody, followed by incubation with an avidin-biotin peroxidase-conjugated secondary antibody, signal developed with diaminobenzidine, and counterstained with hematoxylin. Negative controls were run during each staining procedure. Routine hematoxylin and eosin $(\mathrm{H} \& \mathrm{E})$ staining was performed on each tissue section.

MVD and mitotic figures were compared between the Hyb-PEDF and Hyb- $\beta$ gal experimental groups. Using anti-factor VIII related antigen antibody stained sections; the number of endothelial-lined vessels within the tumor were counted in $5 \mathrm{hpf}$ by two independent reviewers. A brown stain was considered positive and the values were expressed as mean number of vessels per hpf \pm SEM. Using H\&E-stained tissue, mitotic figures were assessed in $5 \mathrm{hpf}$ and values expressed as mean number of mitoses per hpf \pm SEM. VEGF staining intensity was graded on a scale of 1 (absent to minimal staining) to 4 (intense staining) and compared between treatment groups.

Statistical analysis. Statistical significance of data were determined by using unpaired $t$ tests; $p$ values of $<0.05$ were considered statistically significant.

\section{RESULTS}

PEDF secreted by hepatoblastoma cells is a hypoxiasensitive and functional angiogenic mediator. To determine whether the human hepatoblastoma cell line, HUH-6, secreted PEDF, serum-free CM were analyzed by immunoblot. Under normoxic conditions, the HUH-6 cells secreted PEDF as evidenced by the intense band at $50 \mathrm{kD}$ (Fig. 1A, lane 2). However, when HUH-6 cell CM were collected in the presence of $100 \mu \mathrm{M}$ cobalt chloride, a chemical treatment that

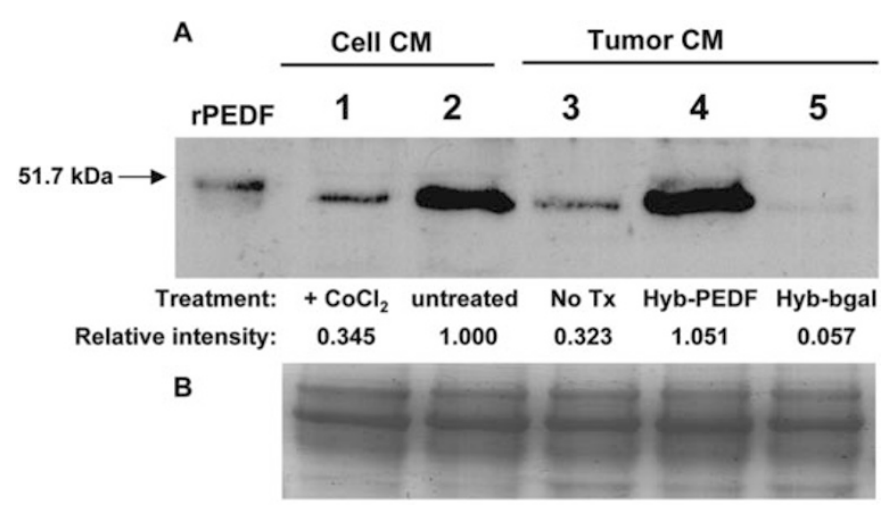

Figure 1. PEDF expressed by hepatoblastoma cells was down-regulated by chemically induced hypoxia and by growth in vivo. Equal amounts of $\mathrm{CM}$ protein $(5 \mu \mathrm{g})$ were subjected to immunoblot analysis for PEDF $(A)$. Recombinant PEDF (rPEDF) was included as a positive control. HUH-6 cells grown in vitro express PEDF (lane 2) and when secretions are collected in the presence of cobalt chloride, a chemical mimetic of hypoxia, there was a 3-fold decrease in PEDF (lane 1). Secretions of minced HUH-6 tumors that received either no treatment (No Tx, lane 3), or were treated with Hyb- $\beta$ gal (lane 5) demonstrated low levels of PEDF compared with that present in equal amounts of the parental cell CM (lane 2). Hyb-PEDF-treated tumor CM contains high levels of PEDF (lane 4). Relative band intensities compared with HUH-6 conditioned media collected from cells (lane 2), as determined by densitometry, are shown below panel $A$. Coomassie-stained gel is shown to demonstrate equal protein loading between lanes $(B)$. 
mimics hypoxic conditions (23), PEDF levels were decreased by $65 \%$ compared with untreated (Fig. 1 A, lane 1 versus lane 2). To assess whether the PEDF secreted by the hepatoblastoma cells was a functional angiogenesis inhibitor, CM collected under normoxic conditions from HUH-6 cells was examined using an in vitro microvascular endothelial cell migration assay, an assay that correlates well with in vivo angiogenic activity (23). The secretions from the HUH-6 cells were angiogenic as determined by their ability to induce endothelial migration 4-fold over the BSA baseline control (Fig. 2). To assess whether VEGF was an active inducer secreted by HUH-6 cells, we added an anti-VEGF antibody, which resulted in a decrease in endothelial cell migration (Fig. 2), however, these results did not reach statistical significance, suggesting that other inducers also contribute to the high angiogenic activity. However, addition of an antibody that neutralizes the angio-inhibitory activity of PEDF resulted in a significant increase in cell migration $(p<0.05)$, thus demonstrating that the PEDF secreted by HUH-6 cells does function as an angiogenesis inhibitor.

Gene transfer of PEDF suppresses tumor growth. Subcutaneous tumors were induced in athymic mice using $3 \times 10^{6}$ HUH- 6 cells. All animals thrived during the treatment period. Animals bearing tumors $5 \mathrm{~mm}$ in diameter were randomized into treatment groups. There was no significant difference in tumor volumes between those tumors receiving no treatment

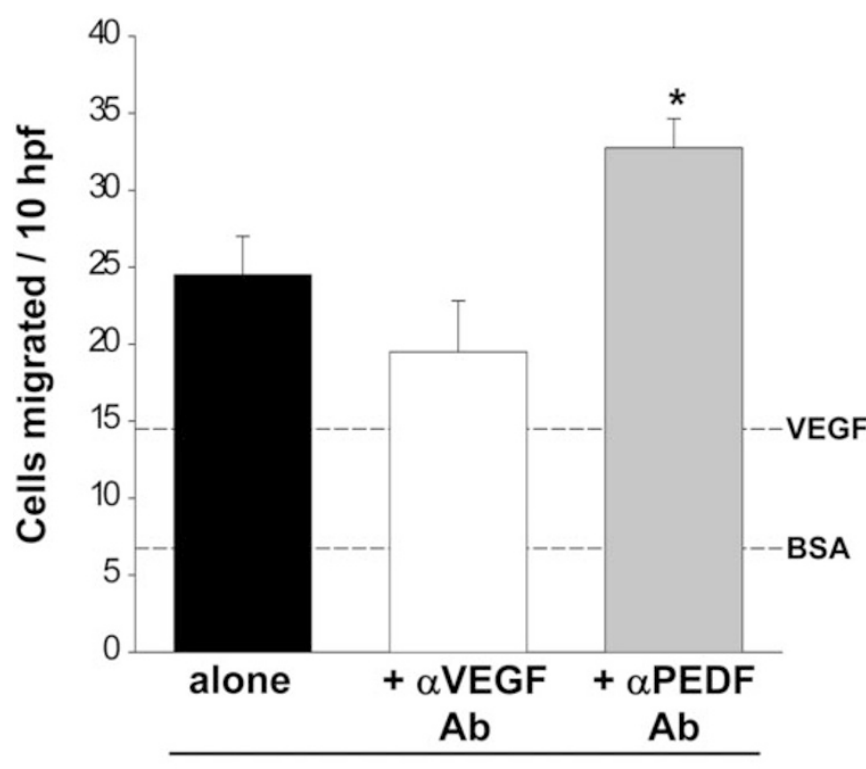

HUH- 6 conditioned media

Figure 2. Endothelial migration was stimulated by secretions of HUH-6 cells. When tested alone, the conditioned media of HUH-6 cells (black bar) had proangiogenic activity as indicated by their ability to induce endothelial migration 4-fold over the baseline control, DMEM + 0.1\% BSA alone (BSA, lower dashed line). Purified VEGF is included as a positive control in DMEM $+0.1 \%$ BSA (VEGF, upper dashed line). The reduction of angiogenic activity by the addition of neutralizing VEGF antibody $(+\alpha \mathrm{VEGF} \mathrm{Ab}$; white bar) was not statistically significant, suggesting the presence of additional inducers. The addition of antibody that neutralized the inhibitory activity of PEDF $(+\alpha$ PEDF Ab; gray bar $)$ resulted in a significantly increased migration ( $p<0.05 ; n=4$ samples assayed per group), demonstrating the importance of endogenous PEDF as a barrier to hepatoblastoma angiogenesis. and those treated with Hyb- $\beta$ gal ( $p=0.52$; Fig. 3 ). In contrast, tumors treated with Hyb-PEDF had a significantly lower tumor volume compared with the Hyb- $\beta$ gal treated tumors (d 7 mean $=97.67 \pm 9.8$ versus $248 \pm 28.3 ; p<0.008$, Fig. 3 ). Histologic examination revealed that the tumors treated with Hyb-PEDF had more differentiated tumor cells characterized by diminished nuclear to cytoplasmic ratios and a tendency to form trabecula. Hyb-PEDF tumors also had confluent areas of necrosis. In contrast, the tumors harvested for the Hyb- $\beta$ gal treatment group had sheets of pleomorphic tumor cells with atypical mitoses and minimal to no necrosis.

To compare the levels of PEDF produced by the untreated and Hyb- $\beta$ gal- and Hyb-PEDF-treated tumors, tumor tissue was minced, and CM was collected and evaluated by immunoblot analysis. Interestingly, the levels of PEDF produced by the untreated and the Hyb- $\beta$ gal treated tumors were lower than what would have been predicted by the level produced by the parental cells, at $\sim 32 \%$ and $6 \%$ the level of the cells grown in vitro (Fig. 1, compare lanes 3 and 5 to lane 2), suggesting that growth in vivo selected for HUH-6 cells secreting low levels of PEDF or that the in vivo growth conditions down-regulate PEDF expression. The CM of the Hyb-PEDF treated tumors expressed levels equivalent to the cells grown in vitro and $\sim 3$-fold higher levels of PEDF than the untreated tumors and almost 20-fold higher than the Hyb- $\beta$ gal tumor CM (Fig. 1, compare lane 4 to lane 5), demonstrating that PEDF expression was higher and persistent in the Hyb-PEDF tumors. The uptake of the virus in the Hyb- $\beta$ gal-treated tumors was verified by $\beta$-galactosidase staining (data not shown).

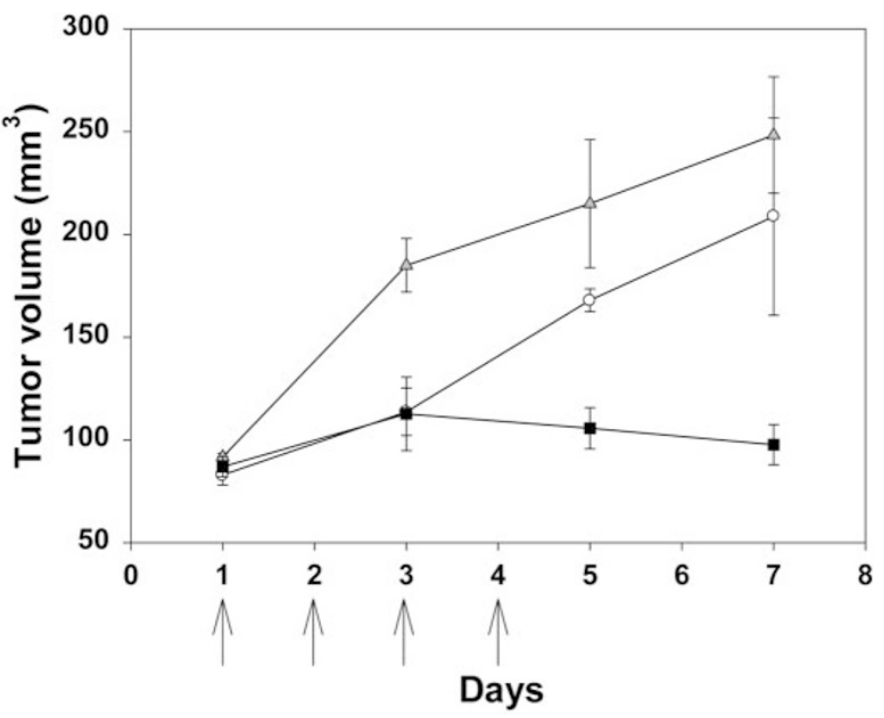

Figure 3. Xenograft hepatoblastoma tumor growth was significantly reduced by treatment with Hyb-PEDF. HUH-6 induced tumors in athymic mice were measured and volumes calculated. Graph symbols are as follows: untreated (white circles); Hyb- $\beta$ gal treated (gray triangles); and, Hyb-PEDF treated (black squares). Tumors treated with the Hyb-PEDF viral vector had a significantly lower tumor volume compared with the control Hyb- $\beta$ gal treated tumors ( $p<0.008 ; n=4$ mice/group). Arrows indicate daily injections of viral vectors. 
Hyb-PEDF treatment altered tumor endothelial and epithelial cells. To assess whether the decrease in tumor growth resulted from inhibition of angiogenesis, MVD was determined. MVD was significantly higher in the Hyb- $\beta$ gal group compared with the Hyb-PEDF treatment group (15.7 \pm 0.61 versus $4.4 \pm 0.39, p<0.001$, Fig. $4, A-C$ ). In addition, a 4-fold higher number of mitotic figures was noted in the tumors treated with Hyb- $\beta$ gal compared with the Hyb-PEDF treatment group $(17.0 \pm 0.80$ versus $4.4 \pm 0.37, p<0.001$, Fig. 5, $A-C$ ). PCNA staining showed a similar fold decrease in the Hyb-PEDF group (data not shown). These results suggest that altering the vasculature of a tumor affected the proliferative capacity of the tumor epithelial cells.

Hyb-PEDF treatment resulted in decreased VEGF expression. To determine whether changes in PEDF might affect other hypoxia-sensitive angiogenic mediators such as VEGF, immunohistochemistry for PEDF and VEGF expression was performed on tumor tissue from each group. As expected, PEDF staining was most intense in the Hyb-PEDF treatment group with strong positivity within tumor cells and in the surrounding interstitial tissue. This was in contrast to the weak staining for PEDF observed in the Hyb- $\beta$ gal-treated tumors (Fig. 6, $A$ and $B$ ). Staining with anti-VEGF antibodies also revealed striking differences between the treatment groups. This staining was quantified by grading on a scale of $1-4$. There was intense positivity for VEGF in the Hyb- $\beta$ galtreated tumors, with a mean staining value of $2.75 \pm 0.47$ $(n=4)$, and weak staining for the VEGF in the Hyb-PEDFtreated tumors, with a mean staining value of $1.25 \pm 0.25$
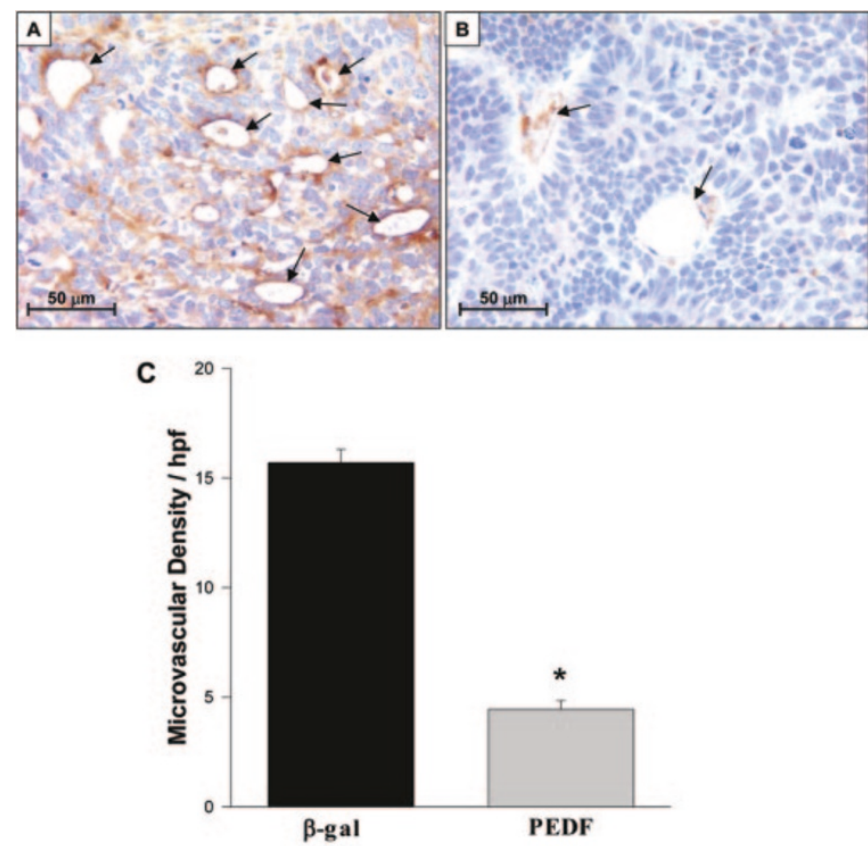

Figure 4. Treatment with Hyb-PEDF resulted in lower microvessel density in xenograft hepatoblastomas. Histologic sections of HUH-6 tumors treated with (A) Hyb- $\beta$ gal or $(B)$ Hyb-PEDF were stained with anti-factor VIII related antigen (brown chromogen). Arrows indicate blood vessels within the tumor sections. Microvessel density was determined $(C)$, and the Hyb- $\beta$ gal control group had a 4-fold higher MVD than the Hyb-PEDF treatment group $(p<$ $0.001 ; n=4$ mice/group). Magnification: $40 \times$.
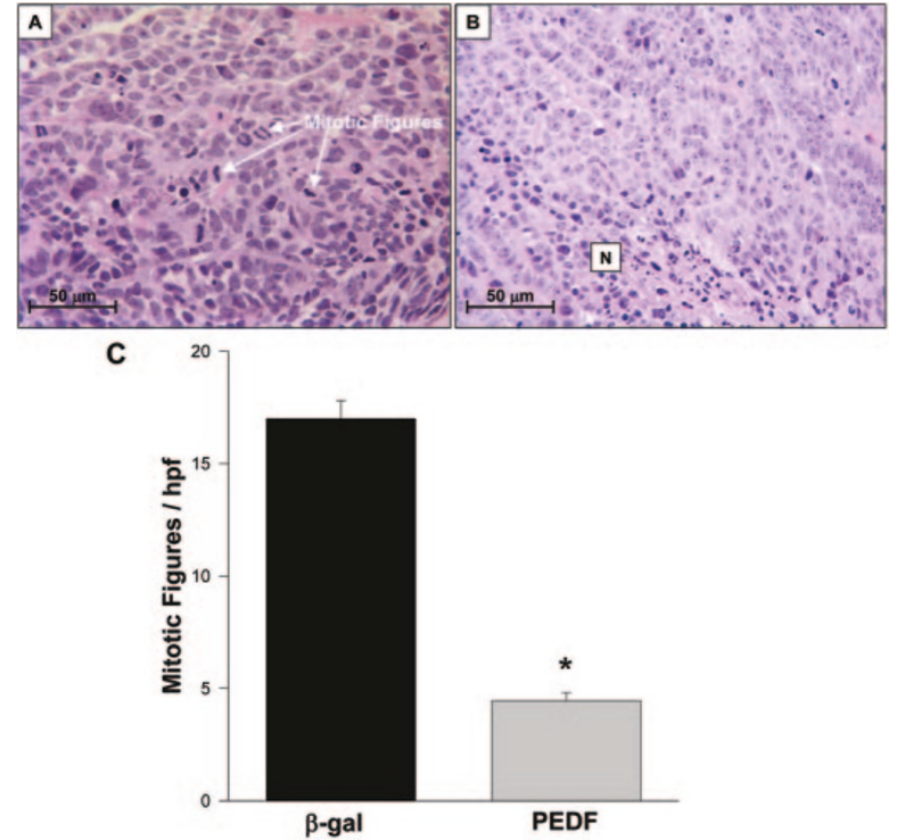

Figure 5. Treatment of HUH-6 tumors with Hyb-PEDF resulted in decreased number of mitotic figures. Mitotic figures were counted in H\&E-stained sections of HUH-6 tumors that were treated with either $(A)$ Hyb- $\beta$ gal or with (B) Hyb-PEDF. The mitotic figure count was increased by 4 -fold in the Hyb- $\beta$ gal group compared with the Hyb-PEDF treatment group $(C ; p<$ $0.001 ; n=4$ mice/group). In panel $B$, "N" denotes an area of necrosis. Magnification: $40 \times$.
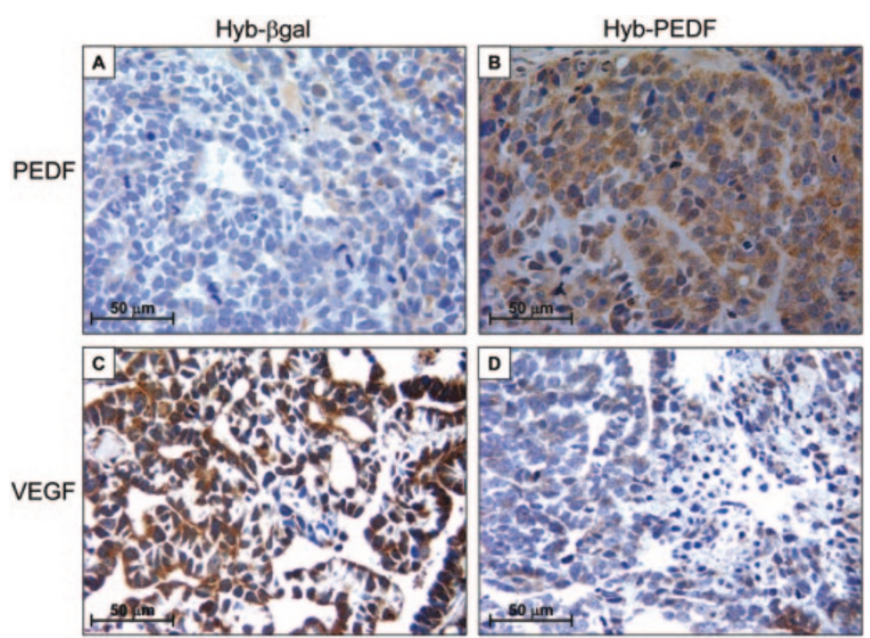

Figure 6. Immunohistochemical staining of HUH-6 tumors for PEDF and VEGF. PEDF staining in Hyb- $\beta$ gal-treated tumor sections was minimal (A) while in the Hyb-PEDF-treated tumor sections, staining was intense (brown chromogen; panel $B$ ). VEGF staining was strong (brown chromogen) in HUH-6 tumors treated with Hyb- $\beta$ gal $(C)$, compared with the weak VEGF expression in the Hyb-PEDF-treated tumors $(C)$. Sections were counterstained with hematoxylin. Magnification: $40 \times$.

$(n=4 ; p<0.03$; Fig. $6, C$ and $D)$. This suggests that PEDF treatment may directly or indirectly down-regulate VEGF in vivo.

\section{DISCUSSION}

Hepatoblastoma, like most solid tumors, is dependent on pathologic neovascularization for growth and metastasis (27). In this study, we demonstrated a loss of a potent inhibitor of 
angiogenesis, PEDF, in hepatoblastoma in vivo. This study established that forced overexpression of PEDF via gene transfer was able to limit experimental tumor growth. Decreased levels of VEGF and reduced microvascularity may be two mechanisms underlying the altered growth. We found that PEDF expression by human hepatoblastoma cell line (HUH-6) was dramatically decreased under hypoxic conditions and in vivo growth by hepatoblastoma correlated with lower levels of PEDF. Additionally, the baseline angiogenic activity of the HUH-6 was significantly increased when exposed to an antiPEDF antibody suggesting that reduction or loss of endogenous PEDF removes a barrier to growth of the tumor vasculature.

PEDF is a $50 \mathrm{kD}$ multifunctional protein that is found at high levels in the retina, placenta, prostate, kidney, and liver $(28,29)$. Initially labeled as a neurotrophic and neuroprotective factor, PEDF has been recognized as one of the most potent anti-angiogenic factors (15), with a demonstrated ability to inhibit the growth of several cancers in vivo (30-33). As an endogenous inhibitor of angiogenesis, PEDF appears to target only new vessel formation (34) and is stable and nontoxic when administered as a soluble protein or by viral-mediated gene transfer $(30,32,35)$. By only targeting new sprouting vessels, PEDF would not be expected to alter native vasculature. In addition, this type of therapy would not be expected to select for drug-resistance genes that are known to exist in many cancers including hepatoblastoma.

Intratumoral hypoxia can trigger a cascade of tumorpromoting factors. VEGF, HGF, and IGF II are all hypoxiasensitive angiogenic inducers found in hepatoblastoma (2-5). Therapeutic treatment strategies targeting VEGF has been performed with moderate success (16). However, antiangiogenic therapy directed against a single angiogenic inducer may have limited effect due to the presence of numerous inducers within a tumor mass (24). In this study, we demonstrate that PEDF is also hypoxia sensitive in hepatoblastoma, similar to other tumor epithelial cells tested $(20,23)$. The decrease in PEDF expression when tumor cells are exposed to cobalt chloride has also been noted in tumor cell lines derived for hepatocellular carcinoma (33) and prostate cancer (23). This implies that, under hypoxic conditions, a common environmental state within tumor masses, a rise in VEGF can accompany a decrease in PEDF resulting in higher net angiogenic activity. An unexpected benefit of PEDF therapy was decreased expression of VEGF within the hepatoblastoma tumor. VEGF has been demonstrated to play a major role in hepatoblastomas growth and metastasis (2). The ratio of PEDF to VEGF has been shown to be important in angiogenesisbased diseases, with a low ratio seen in retinal disease, melanoma, and neuroblastoma $(32,36,37)$. Our results suggest that a higher PEDF to VEGF ratio restricts hepatoblastoma growth, and suggests that PEDF may regulate neovascularization and tumor growth by suppressing VEGF's activities in addition to acting directly on the endothelial cell.

Gene therapy has been advocated as a potential antiangiogenic therapy delivery system for several reasons (38). It allows for low-frequency dosing with sustained therapy production by the infected cells. Intratumoral gene therapy allows for selective expression by tumor cells resulting in higher local concentrations within the tumor while maintaining lower systemic levels limiting the number of adverse reactions (38). After several consecutive administrations of our hybrid vector, its expression remained high for several days after the last dose. Further studies are needed to assess the effect of alternate dosing schedules, and the persistence of PEDF expression within treated tumors.

Therapeutic replacement of inhibitors such as PEDF has the distinct advantage of counteracting the stimulating endothelial cell activities of a wide array of angiogenic inducers (20). In experimental models, PEDF shows minimal toxicity, spares normal cells from unnecessary death, and targets both endothelial and tumor epithelial cells. Development of new therapeutic avenues with agents like PEDF may provide hope for those children with chemo-resistant or inoperable hepatoblastoma tumors.

\section{REFERENCES}

1. Stocker JT, Schmidt D 2000 Hepatoblastoma. In: Hamilton SR, Aaltonen LA (eds) Pathology and Genetics: Tumors of the Digestive System. Lyon Press, Oxford, pp 184-189

2. Sun XY, Wu ZD, Liao XF, Yuan JY 2005 Tumor angiogenesis and its clinical significance in pediatric malignant liver tumors. World J Gastroenterol 11:741-743

3. von Schweinitz D, Fuchs J, Gluer S, Pietsch T 1998 The occurrence of liver growth factor in hepatoblastoma. Eur J Pediatr Surg 8:133-136

4. Kim MS, Lee YM, Moon EJ, Kim SE, Lee JJ, Kim KW 2000 Anti-angiogenic activity of torilin, a sesquiterpene compound isolate from Torilis japonica. Int $\mathrm{J}$ Cancer 87:269-275

5. Kim KR, Moon HE, Kim KW 2002 Hypoxia-induced angiogenesis in human hepatocellular carcinoma. J Mol Med 80:703-714

6. Shweiki D, Itin A, Soffer D, Keshet E 1992 Vascular endothelial growth factor induced by hypoxia may mediate hypoxia-initiated angiogenesis. Nature 359:843845

7. Plate KH, Breier G, Weich HA, Risau W 1992 Vascular endothelial growth factor is a potential tumour angiogenesis factor in human gliomas in vivo. Nature 359:845848

8. Pennacchietti S, Michieli P, Galluzzo M, Mazzone M, Giordano S, Comoglio PM 2003 Hypoxia promotes invasive growth by transcriptional activation of the met protooncogene. Cancer Cell 3:347-361

9. Corpechot C, Barbu V, Wendum D, Chignard N, Housset C, Poupon R, Rosmordue O 2002 Hepatocyte growth factor and c-Met inhibition by hepatic cell hypoxia: a potential mechanism for liver regeneration failure in experimental cirrhosis. Am J Pathol 160:613-620

10. Fukuoka H, Aoyama M, Miyazawa K, Asai K, Goto S 2005 Hypoxic stress enhances osteoclast differentiation via increasing IGF-2 production by non-osteoclastic cells. Biochem Biophys Res Commun 328:885-894

11. Augustin HG 2003 Translating angiogenesis research into the clinic: the challenges ahead. Br J Radiol 76:S3-S10

12. Shimizu K, Oku N 2004 Cancer anti-angiogenic therapy. Biol Pharm Bull 27:599605

13. Purow B, Fine HA 2004 Progress report on the potential of angiogenesis inhibitors for neuro-oncology. Cancer Invest 22:577-587

14. von Schweinitz D, Hecker H, Harms D, Bode U, Weinel P, Burger D, Erttmann R, Mildenberger H 1995 Complete resection before development of drug resistance is essential for survival form advanced hepatoblastoma-a report from the German cooperative pediatric liver tumor study HB-89. J Pediatr Surg 30:845-852

15. Boehm T, Folkman J, Browder T, O'Reilly MS 1997 Antiangiogenic therapy of experimental cancer does not induce acquired drug resistance. Nature 390:404-407

16. McCrudden KW, Hopkins B, Frischer J, Novikov A, Huang J, Kadenhe A, New T, Yokoi A, Yamashiro DJ, Kandell JJ, Middlesworth W 2003 Anti-VEGF antibody in experimental hepatoblastoma: suppression of tumor growth and altered angiogenesis. J Pediatr Surg 38:308-314

17. Matsusaka S, Nakasjo K, Terada N, Sugihara A, Tsujimura T, Takanashi T, Uematsu K, Okamoto E, Toyosaka A 2000 Inhibition by an angiogenesis inhibitor, TNP-470, of the growth of a human hepatoblastoma heterotransplanted into nude mice. J Pediatr Surg 35:1198-1204

18. Bouck N, Stellmach V, Hsu SC 1996 How tumors become angiogenic. Adv Cancer Res 69:135-174

19. Hanahan D, Folkman J 1996 Patterns and emerging mechanisms of the angiogenic switch during tumorigenesis. Cell 86:353-364

20. Dawson DW, Volpert OV, Gillis P, Crawford SE, Xu H, Benedict W, Bouck NP 1999 Pigment epithelium-derived factor: a potent inhibitor of angiogenesis. Science 285:245-248

21. Tombran-Tink J, Mazuruk K, Rodriguez IR, Chung D, Linker T, Englander E, Chader GJ 1996 Organization, evolutionary conversation, expression and unusual 
Alu density of the human gene for pigment epithelium-derived factor, a unique neurotrophic serpin. Mol Vis 2:11-

22. Sawant S, Aparicio S, Tink AR, Lara N, Barnstable CJ, Tombran-Tink J 2004 Regulation o factors controlling angiogenesis in liver development: a role for PEDF in the formation and maintenance of normal vasculature. Biochem Biophys Res Commun 325:408-413

23. Doll JA, Stellmach VM, Bouck NP, Berg AR, Lee C, Abramson LP, Cornwell ML, Pins MR, Borensztajn J, Crawford SE 2003 Pigment epithelium-derived factor regulates the vasculature and mass of the prostate and pancreas. Nat Med 9:774-780

24. Doll JA, Reiher FK, Crawford SE, Pins MR, Campbell SC, Bouck NP 2001 Thrombospondin-1, vascular endothelial growth factor and fibroblast growth factor-2 are key functional regulators of angiogenesis in the prostate. Prostate 49:293305

25. Lee EJ, Anderson LM, Thimmapaya B, Jameson JL 1999 Targeted expression of toxic genes directed by pituitary hormone promoters: a potential strategy for adenovirus-mediated gene therapy of pituitary tumors. J Clin Endocrinol Metab 84:786-794

26. Ghaneh P, Greenhalf W, Humphreys M, Wilson D, Zumstein L, Lemoine NR, Neoptolemus JP 2001 Adenovirus-mediated transfer of $\mathrm{p} 53$ and $\mathrm{p} 16^{\mathrm{INK} 4 \mathrm{a}}$ results in pancreatic cancer regression in vitro and in vivo. Gene Ther 8:199-208

27. Folkman J 1990 What is the evidence that tumors are angiogenesis dependent? J Natl Cancer Inst 82:4-6

28. Tombran-Tink J, Barnstable CJ 2003 PEDF: multifaceted neurotrophic factor. Nat Rev Neurosci 4:628-636

29. Chader GJ 2001 PEDF: raising both hopes and questions in controlling angiogenesis. Proc Natl Acad Sci U S A 98:2122-2124

30. Abramson LP, Stellmach V, Doll JA, Cornwell M, Arensman RM, Crawford SE 2003 Wilms' tumor growth is suppressed by anti-angiogenic pigment epitheliumderived factor in xenograft model. J Pediatr Surg 38:336-342
31. Crawford SE, Stellmach V, Ranalli M, Huang X, Huang L, Volpert O, De Vries GH, Abramson LP, Bouck N 2001 Pigment epithelium-derived factor (PEDF) in neuroblastoma: a multifunctional mediator of Schwann cell antitumor activity. J Cell Sci 114:4421-4428

32. Streck CJ, Zhang Y, Zhou J, Ng C, Nathwani AC, Davidoff AM 2005 Adenoassociated virus vector-mediated delivery of pigment epithelium-derived factor restricts neuroblastoma angiogenesis and growth. J Pediatr Surg 40:236-243

33. Matsumoto K, Ishikawa H, Nishimura D, Hamasaki K, Nakao K, Eguchi K 2004 Antiangiogenic property of pigment epithelium-derived factor in hepatocellular carcinoma. Hepatology 40:252-259

34. Stellmach V, Crawford SE, Zhou W, Bouck N 2001 Prevention of ischemia-induced retinopathy by the natural ocular antiangiogenic agent pigment epithelium-derived factor. Proc Natl Acad Sci U S A 98:2593-2597

35. Mahtabifard A, Merritt RE, Yamada RE, Crystal RG, Korst RJ 2003 In vivo gene transfer of pigment epithelium-derived factor inhibits tumor growth in syngeneic murine models of thoracic malignancies. J Thorac Cardiovasc Surg 126:28-38

36. Duh EJ, Yang HS, Suzuma I, Miyagi M, Youngman E, Mori K, Katai M, Yan L, Suzuma K, West K, Davarya S, Tong P, Gehlbach P, Pearlman J, Crabb JW, Aiello LP, Campochiaro PA, Zack DJ 2002 Pigment epithelium-derived factor suppresses ischemia-induced retinal neovascularization and VEGF-induced migration and growth. Invest Ophthamol Vis Sci 43:821-829

37. Garcia M, Fernandez-Garcia NI, Rivas V, Carretero M, Escamez MJ, GonzalezMartin A, Medrano EE, Volpert O, Jorcano JL, Jimenz B, Larcher F, Del Rio M 2004 Inhibition of xenografted human melanoma growth and prevention of metastasis development by dual antiangiogenic/antitumor activities of pigment epitheliumderived factor. Cancer Res 64:5632-5642

38. Lau K, Bicknell R 1999 Antiangiogenic gene therapy. Gene Ther 6:1793-1795 